\title{
A Brain-Computer Music Interface for Music Expression
}

\author{
Rafael Ramirez, Sergio Giraldo, Zacharias Vamvakousis \\ Music Technology Group, Universitat Pompeu Fabra, Barcelona, Spain \\ rafael.ramirez@upf.edu
}

\begin{abstract}
Active music listening is a way of listening to music through active interactions. In this paper we present an expressive brain-computer interactive music system for active music listening, which allows listeners to manipulate expressive parameters in music performances using their emotional state, as detected by a braincomputer interface. The proposed system is divided in two parts: a real-time system able to detect listeners' emotional state from their EEG data, and a real-time expressive music performance system capable of adapting the expressive parameters of music based on the detected listeners' emotion. We comment on an application of our system as a music neurofeedback system to alleviate depression in elderly people.
\end{abstract}

Keywords: Brain-computer interfaces, emotion, expressive music performance

\section{Introduction}

In recent years, active music listening has emerged as a study field that aims to enable listeners to interactively control different aspects of music in real-time. Most of the work in this area has focused on controlling music aspects such as playback, equalization, browsing and retrieval. However, there have been very few attempts to control the expressive aspects of music performance, such as pitch, timing, amplitude and timbre. The manipulation of these sound properties is clearly distinguishable by the listeners who often experience strong emotions as a consequence of this. Expressive music performance research (for an overview see Gabrielsson, 1999, 2003) investigates the manipulation of these sound properties in an attempt to understand how it conveys emotion and to recreate expression in performances.

The study of emotions in human-computer interaction has increased in recent years. This is due to the growing need for computer applications capable of detecting the emotional state of users (Picard, 2002). Motivated by every day interaction among humans, a great part of the research in this area has explored detecting emotions from facial and voice information. Under controlled conditions, current emotion-detection computer systems based on such information are able to classify emotions with considerable accuracy (Takahashi, 2004). However, emotions are not always manifested by means of facial expressions and voice information. Facial and voice information is related only to behavioral expression, which can be consciously controlled and modified, and which interpretation is often subjective. A still relatively new field of research in affective brain-computer interaction attempts to detect a person's emotional state using electroencephalograms (EEGs) (Chanel, 2006; Choppin, 2000).

In this paper we go one step beyond brain activity data sonification by mapping brain activity data into a high-level emotional space description, and use this description to control expressive aspects of music performances. The result is an expressive brain-computer interac- 
tive music system for active music listening, which allows listeners to manipulate expressive parameters in music performances using their emotional state, as detected by a braincomputer interface. We describe the application of our system as a music neurofeedback system to alleviate depression in elderly people.

\section{A Brain-Computer Music Interface for Music Expression}

Our approach to emotion-based expressive brain-computer music interaction is depicted in Figure 1. The system consists of a real-time feedback loop in which the brain activity of a person is captured as an EEG signal, the signal is processed in order to filter some frequency bands and to compute the estimated emotional state of a person as a coordinate in the arousal-valence $2 \mathrm{D}$ space. This coordinate, together with a music score or audio file, is the input to a previously trained expressive music performance model, which outputs an expressive rendition of the score/audio. The expressive performance output is presented to the user as feedback of his/her emotional state.

For acquiring the brain activity of the user, we use the Emotiv EPOC headset, recently released by the Emotiv Company (Emotiv, 2014). This headset consists of 14 datacollecting electrodes and 2 reference electrodes, located and labeled according to the international 10-20 system (Niedermeyer, 2004).

From the EEG signal of a person, we determine the level of arousal, i.e. how relaxed or excited the person is, by computing the ratio of the beta and alpha brainwaves as recorded by the EEG. In order to determine the valence level, i.e. negative or positive state of mind, we compare the activation levels of the two cortical hemispheres. Details about arousal and valence calculation can be found in Ramirez et al. 2012.

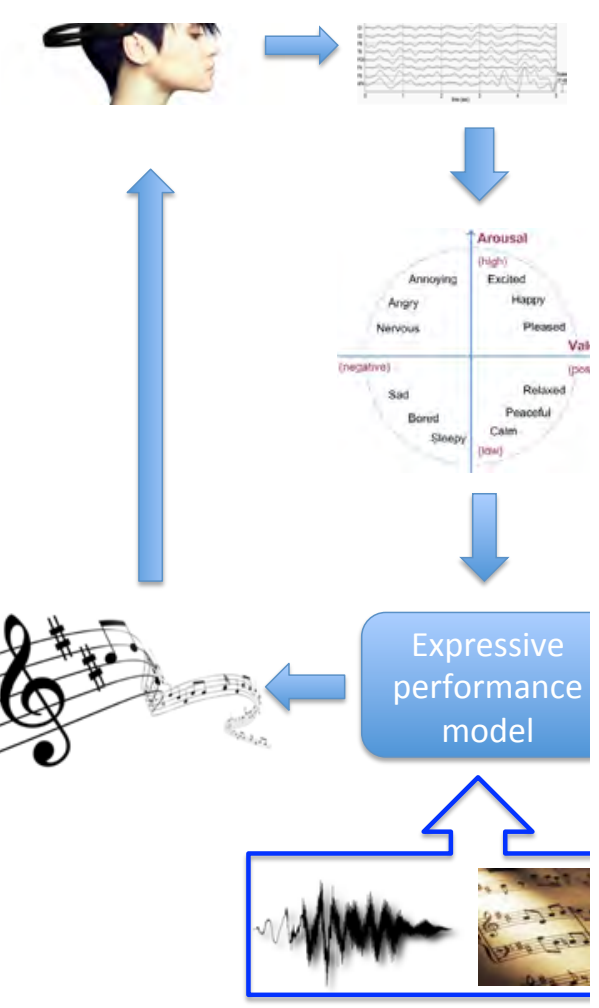

Figure 1. System overview

We trained four expressive performance models (one for each emotion, happy, sad, angry and tender) using machine learning techniques. In order to train the model we have collected a training set of music scores of pieces and recorded performances of the pieces in the four different emotions by a professional musician. The modelled expressive performance actions are duration ratio (ratio between the score duration and the performed duration), the energy ratio (ratio between loudness of a note and average loudness), and articulation ratio (level of staccato - legato).

In order to apply the expressive model with an arousal-valence coordinate as input, we interpolate (using a linear regression model) the four trained (happy, sad, angry and tender) models. This allows us to apply intermediate models, not just the four trained models. The performance actions, i.e. duration, energy and articulation, are calculated using the model and the interpolated coefficients based on a control position input on the arousal valence plane. 


\section{A music neurofeedback application}

The proposed expressive brain-computer music interface has been applied as a music neurofeedback system for treating depression in elderly people. Ten adults (nine female and one male, mean $=84, S D=5.8$ ) with normal hearing participated in the neurofeedback study consisting of ten sessions (two sessions per week) of fifteen minutes each. Participants listened to music pieces preselected according to their music preferences, and were encouraged to increase the loudness and tempo of the pieces, based on their arousal and valence levels, respectively: Pre and post evaluation of six participants was performed using the BDI depression test, showing an average improvement of $17.2 \%$ in their BDI scores at the end of the study.

\section{Conclusions}

We have presented an expressive braincomputer interactive music system for active music listening, which allows listeners to manipulate expressive parameters in music performances using their emotional state, as detected by their brain activity as an EEG signal. The proposed system is divided in two parts: a real-time system able to detect listeners' emotional state from their EEG data, and a realtime expressive music performance system capable of adapting the expressive parameters of music based on the detected listeners' emotion. For acquiring the brain activity of the listener, we use the low-cost Emotiv EPOC headset, and we determine his/her emotional estate by computing arousal and valence values from the alpha and beta waves in the prefrontal cortex. The expressive performance model is trained using recordings of musical pieces in four emotions (happy, relaxed, sad, and angry). The input of the system is the coordinate consisting of the computed instantaneous arousal and valence values. The coefficients of the four expressive models (one for each emotion) are interpolated to obtain the prediction of the performance actions, which serve as input for synthesis. Finally, we have briefly described the application of the proposed system as a music neurofeedback system to alleviate depression in elderly people.

\section{Acknowledgements}

This work has been partly sponsored by the Spanish TIN project TIMUL (TIN2013-48152(2-2-R)

\section{References}

Chanel, G., Kronegg, J., Grandjean, D., Pun, T.: Emotion Assessment: Arousal Evaluation Using EEG's and Peripheral Physiological Signals. In: Gunsel, B., Jain, A.K., Tekalp, A.M., Sankur, B. (eds.) MRCS 2006. LNCS, vol. 4105, pp. 530-537. Springer, Heidelberg (2006)

Choppin, A.: Eeg-based human interface for disabled individuals: Emotion expression with neural networks. Masters thesis, Tokyo Institute of Technology, Yokohama, Japan (2000)

Emotiv Systems Inc. Researchers, (2014). URL http://www.emotiv.com/researchers/.

Gabrielsson, A. The performance of music. In: Deutsch, D. (Ed.), The Psychology of Music, second ed. Academic Press (1999).

Gabrielsson, A. Music performance research at the millennium. Psychol. Music 31 (3), 221-272 (2003)

Niedermeyer, E., da Silva, F.L.: Electroencephalography, Basic Principles, Clinical Applications, and Related Fields, p. 140. Lippincott Williams \& Wilkins (2004)

Picard, R.W., Klein, J.: Toward computers that recognize and respond to user emotion: Theoretical and practical implications. Interacting with Computers 14(2), 141-169 (2002)

Ramirez, R., Vamvakousis, Z. (2012). Detecting Emotion from EEG Signals Using the Emotive Epoc Device, Brain Informatics, LNCS 7670, pp. 175-184.

Takahashi, K.: Remarks on emotion recognition from bio-potential signals. In: 2nd International Conference on Autonomous Robots and Agents, pp. 186-191 (2004) 